\title{
ASYMPTOTIC BEHAVIOUR OF LIE POWERS AND LIE MODULES
}

\author{
ROGER M. BRYANT, KAY JIN LIM, AND KAI MENG TAN
}

\begin{abstract}
Let $V$ be a finite-dimensional $F G$-module, where $F$ is a field of prime characteristic $p$ and $G$ is a group. We show that, when $r$ is not a power of $p$, the Lie power $L^{r}(V)$ has a direct summand $B^{r}(V)$ which is a direct summand of the tensor power $V^{\otimes r}$ and which satisfies $\operatorname{dim} B^{r}(V) / \operatorname{dim} L^{r}(V) \rightarrow 1$ as $r \rightarrow \infty$. Similarly, for the same values of $r$, we obtain a projective submodule $C(r)$ of the Lie module Lie $(r)$ over $F$ such that $\operatorname{dim} C(r) / \operatorname{dim} \operatorname{Lie}(r) \rightarrow 1$ as $r \rightarrow \infty$.
\end{abstract}

\section{INTRODUCTION}

Let $F$ be a field of prime characteristic $p, G$ a group, and $V$ a finite-dimensional (right) $F G$-module. Write $T(V)$ for the tensor algebra of $V$ : thus $T(V)=\bigoplus_{r=0}^{\infty} V^{\otimes r}$. Let $L(V)$ denote the Lie subalgebra of $T(V)$ generated by $V$ : thus $L(V)=\bigoplus_{r=1}^{\infty} L^{r}(V)$, where $L^{r}(V)=L(V) \cap V^{\otimes r}$. We call $L^{r}(V)$ the $r$ th Lie power of $V$. The tensor power $V^{\otimes r}$ is an $F G$-module (under the 'diagonal' action of $G$ ) and $L^{r}(V)$ is a submodule of $V^{\otimes r}$. The dimension of $L^{r}(V)$ is given by a formula of Witt (see MKS, Theorem 5.11]):

$$
\operatorname{dim} L^{r}(V)=\frac{1}{r} \sum_{d \mid r} \mu(d) n^{r / d},
$$

where $n=\operatorname{dim} V$ and $\mu$ denotes the Möbius function.

The modules $L^{r}(V)$ have been extensively studied in recent years. We refer to the paper of Bryant and Schocker [BS1] and the works cited there for details of progress on the problem of describing $L^{r}(V)$ up to isomorphism. When $r$ is not divisible by $p, L^{r}(V)$ is a direct summand of $V^{\otimes r}$ (see, for example, [DE, §3.1]). Although this does not hold in general, our first main result shows that, in an asymptotic sense, 'most' of $L^{r}(V)$ is a direct summand of $V^{\otimes r}$ provided that $r$ is not a power of $p$.

Theorem 1.1. Suppose that $\operatorname{dim} V>1$. Let $\mathcal{A}$ be the set of all positive integers $r$ such that $r$ is not a power of $p$. Then, for each $r \in \mathcal{A}$, there is a direct summand $B^{r}(V)$ of $L^{r}(V)$ such that $B^{r}(V)$ is a direct summand of $V^{\otimes r}$ and

$$
\lim _{\substack{r \rightarrow \infty \\ r \in \mathcal{A}}} \frac{\operatorname{dim} B^{r}(V)}{\operatorname{dim} L^{r}(V)}=1
$$

The modules $B^{r}(V)$ are the modules denoted by $B_{r}$ in the decomposition theorem of [BS1] and their dimensions are given by a recurrence formula. When $r=p^{m}$ with

Date: November 2010.

2000 Mathematics Subject Classification. 17B01, 20C30 (primary), 20C20 (secondary).

Supported by EPSRC Standard Research Grant EP/G024898/1 and EP/G025487/1, and by MOE Academic Research Fund R-146-000-135-112. 
$m>0$, we have $B_{r}=0$. Thus, although we conjecture that a result like Theorem 1.1 holds without any restrictions on $r$, we cannot use BS1 when $r$ is a power of $p$.

The other main result of this paper concerns the Lie module $\operatorname{Lie}(r)$. This is a module for the symmetric group $\mathfrak{S}_{r}$ and can be defined as follows. Let $E_{n}$ be a vector space over $F$ of finite dimension $n$, where $n \geqslant r$, and let $\left\{e_{1}, \ldots, e_{n}\right\}$ be a basis for $E_{n}$. Then $\operatorname{Lie}(r)$ is the subspace of $L^{r}\left(E_{n}\right)$ spanned by all elements of the form $\left[e_{1 \pi}, e_{2 \pi}, \ldots, e_{r \pi}\right]$ where $\pi \in \mathfrak{S}_{r}$ and $\left[e_{1 \pi}, e_{2 \pi}, \ldots, e_{r \pi}\right]$ denotes the 'leftnormed' Lie product in which $\left[e_{1 \pi}, e_{2 \pi}\right]=e_{1 \pi} \otimes e_{2 \pi}-e_{2 \pi} \otimes e_{1 \pi}$ and $\left[e_{1 \pi}, e_{2 \pi}, \ldots, e_{r \pi}\right]=$ $\left[\left[e_{1 \pi}, e_{2 \pi}, \ldots, e_{(r-1) \pi}\right], e_{r \pi}\right]$. Clearly Lie $(r)$ is a (right) $F \mathfrak{S}_{r}$-module, where the action of $\mathfrak{S}_{r}$ comes from its right action on $\{1, \ldots, r\}$. Furthermore, the definition of Lie $(r)$ is independent of the choice of $n$ with $n \geqslant r$. The dimension of $\operatorname{Lie}(r)$ is $(r-1)$ ! (as follows from [MKS, Theorem 5.11] by taking $n_{1}=n_{2}=\cdots=1$ ).

The main motivation for our result on $\operatorname{Lie}(r)$ comes from the work of Selick and $\mathrm{Wu}$ [SW1]. These authors considered the problem of finding natural homotopy decompositions of the loop suspension of a $p$-torsion suspension and proved that this problem is equivalent to the algebraic problem of finding natural coalgebra decompositions of the primitively generated tensor algebras over the field with $p$ elements. They determined (see [SW1, Theorem 6.5]) the finest coalgebra decomposition of a tensor algebra (over an arbitrary field). Their result can be described as a functorial Poincaré-Birkhoff-Witt theorem. However, in order to compute the factors in this decomposition, it is necessary to know a maximal projective submodule, $\operatorname{Lie}^{\max }(r)$, of the Lie module $\operatorname{Lie}(r)$.

The projective modules for the symmetric groups over fields of positive characteristic $p$ are not known in general. Their structure depends on the decomposition matrices for symmetric groups and the determination of these is a famous open problem. However, according to [SW2, even if the modules Lie ${ }^{\max }(r)$ cannot be computed precisely, it is of interest to know how quickly their dimensions grow and whether or not the growth rate is exponential. Determination of $\mathrm{Lie}^{\max }(6)$ and $\mathrm{Lie}^{\max }(8)$ in characteristic 2 in [SW2] suggests that Lie ${ }^{\max }(r)$ is relatively large compared with $\operatorname{Lie}(r)$. If this is true in general, it has the desirable consequence that the factors in the functorial PBW theorem are relatively small.

Some progress has been made in the understanding of $\operatorname{Lie}^{\max }(r)$ in the case where $r=p k$ with $p \nmid k$. Erdmann and Schocker [ES] established, amongst other things, that there is a one-to-one correspondence between the non-projective indecomposable summands of $\operatorname{Lie}(p k)$ and the indecomposable summands of Lie $(k)$. Erdmann and Tan [ET] provided an upper bound for $\operatorname{dim} \operatorname{Lie}^{\max }(p k)$ and showed that the ratio of this upper bound to $\operatorname{dim} \operatorname{Lie}(p k)$ approaches 1 as $k$ tends to infinity. They conjectured that the same fact holds for $\operatorname{dim} \operatorname{Lie}^{\max }(p k) / \operatorname{dim} \operatorname{Lie}(p k)$.

Our second main result establishes this conjecture and considerably more.

Theorem 1.2. Let $\mathcal{A}$ be the set of all positive integers $r$ such that $r$ is not a power of $p$. Then

$$
\lim _{\substack{r \rightarrow \infty \\ r \in \mathcal{A}}} \frac{\operatorname{dim} \operatorname{Lie}^{\max }(r)}{\operatorname{dim} \operatorname{Lie}(r)}=1 .
$$

The theorem shows, in particular, that $\operatorname{dim} \operatorname{Lie}^{\max }(r)$ grows exponentially with $r$ when $r$ is not a power of $p$. As in the case of Theorem [1.1, we conjecture that the restriction on $r$ can be removed. However, our methods work only for $r \in \mathcal{A}$. In the 
proof, we quickly reduce to the case where $F$ is an infinite field and then consider the $n$-dimensional natural module $E_{n}$ for the general linear group $\mathrm{GL}_{n}(F)$, where $n \geqslant r$. The image of $L^{r}\left(E_{n}\right)$ under the Schur functor is the Lie module Lie $(r)$ (see Section 3). With $B^{r}\left(E_{n}\right)$ as in Theorem 1.1, the image $C(r)$ of $B^{r}\left(E_{n}\right)$ under the Schur functor is a projective submodule of $\operatorname{Lie}(r)$. We describe the dimension of $C(r)$ by means of a recurrence formula and show that $\operatorname{dim} C(r) / \operatorname{dim} \operatorname{Lie}(r) \rightarrow 1$ as $r \rightarrow \infty$ with $r \in \mathcal{A}$.

Theorem 1.1 will be proved in Section 2 and Theorem 1.2 in Section 3 , Throughout this paper $F$ denotes a field of prime characteristic $p$. All of our algebras and vector spaces are taken over $F$, unless otherwise stated, and all tensor products are also taken over $F$. All modules are right modules.

\section{Modular Lie POWERS}

In this section we shall prove Theorem 1.1.

Let $G$ be a group and let $V$ be a finite-dimensional $F G$-module. The decomposition theorem of [BS1] yields, for each positive integer $r$, a certain direct summand $B_{r}$ of $L^{r}(V)$ such that $B_{r}$ is a direct summand of $V^{\otimes r}$. Here we write $B^{r}(V)$ instead of $B_{r}$. We shall use the following result, where we write $p^{i} U$ for the direct sum of $p^{i}$ copies of a module $U$.

Theorem 2.1 ([BS2, Theorem 4.2]). Let $k$ be a positive integer not divisible by $p$. Then, for each non-negative integer $m$, we have

$$
p^{m} B^{p^{m} k}(V) \oplus p^{m-1} B^{p^{m-1} k}(V)^{\otimes p} \oplus \cdots \oplus B^{k}(V)^{\otimes p^{m}} \cong L^{k}\left(V^{\otimes p^{m}}\right) .
$$

For positive integers $n$ and $r$, let

$$
w(n, r)=\frac{1}{r} \sum_{d \mid r} \mu(d) n^{r / d} .
$$

Lemma 2.2. For all $n$ and $r$, we have

$$
n^{r} / r-n^{r / 2} / 2 \leqslant w(n, r) \leqslant n^{r} / r .
$$

Proof. The result is clear if $r=1$, so we assume $r \geqslant 2$. By [ $\underline{\mathbb{R}}$, Theorem 7.1], $r w(n, r)$ is the number of words of length $r$ on an alphabet of cardinality $n$ that cannot be written as a power of a shorter word. Clearly $r w(n, r) \leqslant n^{r}$. If $u$ is a word of length $r$ of the form $v^{d}$, where $d>1$, then $v$ has length at most $r / 2$. Hence, for a given $d$, the number of possibilities for $v$ is at most $n^{r / 2}$. The number of possibilities for $d$ is at most $r / 2$. Therefore $n^{r}-r w(n, r) \leqslant(r / 2) n^{r / 2}$, which gives the required result.

Let $n=\operatorname{dim}(V)$ and suppose that $n \geqslant 2$. By (1.1), we have $\operatorname{dim} L^{r}(V)=w(n, r)$. For each $r \geqslant 1$, we define

$$
b_{r}=\frac{\operatorname{dim} B^{r}(V)}{\operatorname{dim} L^{r}(V)}=\frac{\operatorname{dim} B^{r}(V)}{w(n, r)} .
$$

Since $B^{r}(V)$ is a submodule of $L^{r}(V)$ we have $0 \leqslant b_{r} \leqslant 1$. In order to prove Theorem 1.1. we need to prove that $b_{r} \rightarrow 1$ as $r \rightarrow \infty$ with $r \in \mathcal{A}$. Note that for $r \in \mathcal{A}$ we can write $r=p^{m} k$ where $m \geqslant 0, p \nmid k$ and $k \geqslant 2$. Suppose, for the rest of this section, 
that $m$ and $k$ satisfy these conditions. By direct computations of the dimensions in (2.1), we have

$$
\sum_{i=0}^{m} p^{m-i}\left(b_{p^{m-i} k}\right)^{p^{i}} w\left(n, p^{m-i} k\right)^{p^{i}}=w\left(n^{p^{m}}, k\right) .
$$

For $i=0,1, \ldots, m$, define

$$
a_{i}=\frac{w\left(n, p^{m-i} k\right)^{p^{i}}}{p^{i} w\left(n, p^{m} k\right)} .
$$

Then, on dividing (2.2) by $p^{m} w\left(n, p^{m} k\right)$ and re-arranging, we obtain

$$
b_{p^{m} k}=\frac{w\left(n^{p^{m}}, k\right)}{p^{m} w\left(n, p^{m} k\right)}-\sum_{i=1}^{m} a_{i}\left(b_{p^{m-i} k}\right)^{p^{i}} .
$$

Lemma 2.3. Suppose that $0<s \leqslant i \leqslant m$. Then

$$
\frac{a_{i}}{a_{i-s}} \leqslant p^{-s}\left(\frac{2 p^{s}}{\left(p^{m-i} k\right)^{p^{s}-1}}\right)^{p^{i-s}} \text {. }
$$

Proof. We have

$$
\frac{a_{i}}{a_{i-s}}=\frac{w\left(n, p^{m-i} k\right)^{p^{i}}}{p^{s} w\left(n, p^{m-i+s} k\right)^{p^{i-s}}}=p^{-s}\left(\frac{w\left(n, p^{m-i} k\right)^{p^{s}}}{w\left(n, p^{m-i+s} k\right)}\right)^{p^{i-s}} .
$$

Write $q=p^{m-i+s} k$. Then, by Lemma 2.2 ,

$$
\frac{w\left(n, p^{m-i} k\right)^{p^{s}}}{w\left(n, p^{m-i+s} k\right)} \leqslant \frac{\left(p^{m-i} k\right)^{-p^{s}} n^{q}}{n^{q} / q-n^{q / 2} / 2}=\frac{\left(p^{m-i} k\right)^{-p^{s}} q n^{q / 2}}{n^{q / 2}-q / 2} .
$$

Since $n \geqslant 2$ and $q \geqslant 6$, we have $n^{q / 2} /\left(n^{q / 2}-q / 2\right) \leqslant 2$. Thus

$$
\frac{w\left(n, p^{m-i} k\right)^{p^{s}}}{w\left(n, p^{m-i+s} k\right)} \leqslant 2\left(p^{m-i} k\right)^{-p^{s}} p^{m-i} k p^{s}=\frac{2 p^{s}}{\left(p^{m-i} k\right)^{p^{s}-1}} \text {. }
$$

This gives the required result.

Corollary 2.4. Suppose that $m \geqslant 1$.

(i) For $i=2,3, \ldots, m-1$, we have $a_{i} / a_{i-1} \leqslant 1$.

(ii) We have $a_{1} \leqslant 2 /\left(p^{m-1} k\right)^{p-1}$ and $a_{m} \leqslant 2 / k^{p^{m}-1}$.

Proof.

(i) By Lemma 2.3 with $s=1$, we have

$$
\frac{a_{i}}{a_{i-1}} \leqslant p^{-1}\left(\frac{2 p}{\left(p^{m-i} k\right)^{p-1}}\right)^{p^{i-1}} \leqslant\left(\frac{2}{p^{(m-i)(p-1)}}\right)^{p^{i}-1} \leqslant 1 .
$$

(ii) By Lemma 2.3 with $i=s=1$, we have

$$
a_{1}=\frac{a_{1}}{a_{0}} \leqslant p^{-1} \frac{2 p}{\left(p^{m-1} k\right)^{p-1}}=\frac{2}{\left(p^{m-1} k\right)^{p-1}} .
$$

Similarly, with $i=s=m$,

$$
a_{m}=\frac{a_{m}}{a_{0}} \leqslant p^{-m} \frac{2 p^{m}}{k^{p^{m}-1}}=\frac{2}{k^{p^{m}-1}} .
$$


Lemma 2.5. We have

$$
\frac{w\left(n^{p^{m}}, k\right)}{p^{m} w\left(n, p^{m} k\right)} \geqslant 1-\frac{k}{2 n^{p^{m} k / 2}} .
$$

Proof. By Lemma 2.2, we have

$$
\frac{w\left(n^{p^{m}}, k\right)}{p^{m} w\left(n, p^{m} k\right)} \geqslant \frac{n^{p^{m} k} / k-n^{p^{m} k / 2} / 2}{p^{m} n^{p^{m} k} /\left(p^{m} k\right)}=1-\frac{k}{2 n^{p^{m} k / 2}} .
$$

Proof of Theorem 1.1. Suppose that $m \geqslant 1$. Recall that $b_{r} \leqslant 1$ for all $r$. By (2.3) and Corollary 2.4(i), we have

$$
1 \geqslant b_{p^{m} k} \geqslant w\left(n^{p^{m}}, k\right) /\left(p^{m} w\left(n, p^{m} k\right)\right)-(m-1) a_{1}-a_{m} .
$$

Hence, by Corollary 2.4(ii) and Lemma 2.5,

$$
1 \geqslant b_{p^{m} k} \geqslant 1-\frac{k}{2 n^{p^{m} k / 2}}-\frac{2(m-1)}{\left(p^{m-1} k\right)^{p-1}}-\frac{2}{k^{p^{m}-1}} .
$$

Also, for $m=0$, we have $b_{k}=w(n, k) / w(n, k)=1$. Thus $b_{r} \rightarrow 1$ as $r \rightarrow \infty$ with $r \in \mathcal{A}$.

\section{LIE MODULES}

In this section we shall prove Theorem 1.2 ,

In order to prove the theorem we shall apply the Schur functor to the modules appearing in a special case of the isomorphism (2.1). Since the Schur functor, in its usual form, requires the field $F$ to be infinite, we begin by showing that it is enough to prove Theorem 1.2 in the case where $F$ is infinite.

Let $G$ be a finite group and let $M$ be a finite-dimensional $F G$-module. We write $M^{\text {max }}$ for a maximal projective submodule of $M$. Thus $M^{\max }$ is a maximal projective direct summand of $M$ and is uniquely determined up to isomorphism. Let $K$ be an extension field of $F$. Then $K \otimes M$ and $K \otimes M^{\max }$ are $K G$-modules.

Lemma 3.1. Suppose that $F$ is a splitting field for $G$. Then $K \otimes M^{\max } \cong(K \otimes$ $M)^{\max }$.

Proof. For modules $U$ and $V$, we write $U \mid V$ to denote that $U$ is isomorphic to a direct summand of $V$. Clearly $K \otimes M^{\max }$ is projective and $K \otimes M^{\max } \mid K \otimes M$. Thus $K \otimes M^{\max } \mid(K \otimes M)^{\max }$. Since $F$ and $K$ are splitting fields for $G$, it follows from [HB, Theorem 10.18] that if $P$ is a projective indecomposable $F G$-module then $K \otimes P$ is a projective indecomposable $K G$-module and, furthermore, every projective indecomposable $K G$-module is isomorphic to some such $K \otimes P$. Hence there is a projective $F G$-module $Q$ such that $K \otimes Q \cong(K \otimes M)^{\text {max }}$. In particular, $K \otimes Q \mid K \otimes M$ and it follows, by [HB, Theorem 1.21], that $Q \mid M$. Thus $Q \mid M^{\max }$ and so $(K \otimes M)^{\max } \mid K \otimes M^{\max }$. The result follows.

In Section 1 we defined the $F \mathfrak{S}_{r}$-module Lie $(r)$ for each positive integer $r$. Let Lie $_{K}(r)$ denote the $K \mathfrak{S}_{r}$-module defined in the same way over $K$. Thus $K \otimes \operatorname{Lie}(r) \cong$ $\operatorname{Lie}_{K}(r)$. As in Section 1, let Lie ${ }^{\max }(r)$ be a maximal projective submodule of Lie $(r)$. It is well-known that every field is a splitting field for $\mathfrak{S}_{r}$ (see [J], Theorem 11.5]). Thus, by Lemma 3.1, $K \otimes \mathrm{Lie}^{\max }(r)$ is isomorphic to a maximal projective submodule 
of $\operatorname{Lie}_{K}(r)$. Consequently, if Theorem 1.2 holds over $K$, it holds over $F$. Therefore it suffices to prove Theorem 1.2 in the case where $F$ is infinite. Hence, from now on, we take $F$ to be infinite.

Suppose that $n \geqslant r$ and let $E_{n}$ be the $n$-dimensional natural module for the general linear group $\mathrm{GL}_{n}(F)$ with standard basis $\left\{e_{1}, \ldots, e_{n}\right\}$. We identify $\mathfrak{S}_{r}$ with the subgroup of $\mathrm{GL}_{n}(F)$ consisting of all those elements which permute $\left\{e_{1}, \ldots, e_{r}\right\}$ and fix $e_{r+1}, \ldots, e_{n}$ pointwise.

Let $\Lambda(n, r)$ be the set of all $n$-tuples $\left(\alpha_{1}, \ldots, \alpha_{n}\right)$ of non-negative integers such that $\alpha_{1}+\cdots+\alpha_{n}=r$. The elements of $\Lambda(n, r)$ are called weights. For $t_{1}, \ldots, t_{n} \in$ $F^{\times}=F \backslash\{0\}$ let $d\left(t_{1}, \ldots, t_{n}\right)$ denote the diagonal matrix in $\operatorname{GL}_{n}(F)$ with entries $t_{1}, \ldots, t_{n}$ down the diagonal.

Let $M$ be a finite-dimensional $F \mathrm{GL}_{n}(F)$-module such that $M$ is a homogeneous polynomial module of degree $r$ (see [G]). For $\alpha=\left(\alpha_{1}, \ldots, \alpha_{n}\right) \in \Lambda(n, r)$ the $\alpha$ weight space of $M$ is denoted by $M^{\alpha}$ and is the subspace of $M$ defined by

$$
M^{\alpha}=\left\{u \in M \mid u \cdot d\left(t_{1}, \ldots, t_{n}\right)=t_{1}^{\alpha_{1}} \cdots t_{n}^{\alpha_{n}} u, \forall t_{1}, \ldots, t_{n} \in F^{\times}\right\} .
$$

By [G, (3.2c)], we have

$$
M=\bigoplus_{\alpha \in \Lambda(n, r)} M^{\alpha} .
$$

Let $\omega_{r} \in \Lambda(n, r)$ be defined by $\omega_{r}=(1, \ldots, 1,0, \ldots, 0)$ where there are $r$ entries equal to 1 and $n-r$ entries equal to 0 . For $\sigma \in \mathfrak{S}_{r} \leqslant \operatorname{GL}_{n}(F)$ we have

$$
\sigma d\left(t_{1}, \ldots, t_{n}\right) \sigma^{-1}=d\left(t_{1 \sigma}, \ldots, t_{r \sigma}, t_{r+1}, \ldots, t_{n}\right) .
$$

It follows that $M^{\omega_{r}}$ is invariant under the restriction to $\mathfrak{S}_{r}$ of the action of $\operatorname{GL}_{n}(F)$ on $M$. Hence $M^{\omega_{r}}$ is an $F \mathfrak{S}_{r}$-module.

Let $\bmod _{F}(n, r)$ be the class of all finite-dimensional homogeneous polynomial $F \mathrm{GL}_{n}(F)$-modules of degree $r$ and let $\bmod \left(F \mathfrak{S}_{r}\right)$ be the class of all finite-dimensional $F \mathfrak{S}_{r}$-modules. Let

$$
f_{r}: \bmod _{F}(n, r) \rightarrow \bmod \left(F \mathfrak{S}_{r}\right)
$$

be the map defined by $f_{r}(M)=M^{\omega_{r}}$ for all $M \in \bmod _{F}(n, r)$. This map $f_{r}$ is called the 'Schur functor'. (It is an exact functor between the two module categories: see G, Chapter 6].) It is easy to verify that

$$
f_{r}(M \oplus N)=f_{r}(M) \oplus f_{r}(N)
$$

for all $M, N \in \bmod _{F}(n, r)$. Furthermore, it is clear from the definition of the Lie module in Section 1 that

$$
f_{r}\left(L^{r}\left(E_{n}\right)\right)=\operatorname{Lie}(r)
$$

for all $n$ such that $n \geqslant r$.

Let $r_{1}, \ldots, r_{l}$ be positive integers such that $r_{1}+\cdots+r_{l}=r$. We may regard $\mathfrak{S}_{r}$ as the group of all permutations of a set of cardinality $r$ written as the disjoint union of sets of cardinalities $r_{1}, \ldots, r_{l}$. Thus we may regard $\mathfrak{S}_{r_{1}} \times \cdots \times \mathfrak{S}_{r_{l}}$ as a subgroup of $\mathfrak{S}_{r}$. The following lemma is an immediate consequence of a slightly stronger version given in [DE].

Lemma 3.2 ([DE, $\S 2.5$, Lemma $])$. Let $n \geqslant r$ and let $r_{1}, \ldots, r_{l}$ be positive integers such that $r_{1}+\cdots+r_{l}=r$. For $i=1, \ldots, l$, let $M_{i} \in \bmod _{F}\left(n, r_{i}\right)$. Then

$$
f_{r}\left(M_{1} \otimes \cdots \otimes M_{l}\right) \cong \operatorname{Ind}_{\mathfrak{S}_{r_{1}} \times \cdots \times \mathfrak{S}_{r_{l}}}^{\mathfrak{S}_{r}}\left(f_{r_{1}}\left(M_{1}\right) \otimes \cdots \otimes f_{r_{l}}\left(M_{l}\right)\right) .
$$


Lemma 3.3. Let $n \geqslant q k$ where $q$ and $k$ are positive integers. Then

$$
\operatorname{dim} f_{q k}\left(L^{k}\left(E_{n}^{\otimes q}\right)\right)=(q k) ! / k .
$$

Proof. Let $I(n, q)$ be the set of all ordered $q$-tuples $\left(i_{1}, \ldots, i_{q}\right)$ where $i_{1}, \ldots, i_{q} \in$ $\{1, \ldots, n\}$. For $\theta \in I(n, q)$, where $\theta=\left(i_{1}, \ldots, i_{q}\right)$, write

$$
e_{\theta}=e_{i_{1}} \otimes \cdots \otimes e_{i_{q}} \in E_{n}^{\otimes q} .
$$

Thus $\left\{e_{\theta} \mid \theta \in I(n, q)\right\}$ is a basis for $E_{n}^{\otimes q}$.

The $F \mathrm{GL}_{n}(F)$-module $L^{k}\left(E_{n}^{\otimes q}\right)$ is spanned as a vector space by the elements $\left[e_{\theta_{1}}, \ldots, e_{\theta_{k}}\right]$ with $\theta_{1}, \ldots, \theta_{k} \in I(n, q)$. It is easily verified that each of these elements belongs to some weight space of $L^{k}\left(E_{n}^{\otimes q}\right)$. Thus, by (3.1), $\left(L^{k}\left(E_{n}^{\otimes q}\right)\right)^{\omega_{q k}}$ is spanned by those elements $\left[e_{\theta_{1}}, \ldots, e_{\theta_{k}}\right]$ which belong to it. These are the elements $\left[e_{\theta_{1}}, \ldots, e_{\theta_{k}}\right]$ such that each of $e_{1}, \ldots, e_{q k}$ occurs once and only once among the tensor factors of $e_{\theta_{1}}, \ldots, e_{\theta_{k}}$.

Let $\Phi$ be the set of all $k$-element subsets $\left\{\theta_{1}, \ldots, \theta_{k}\right\}$ of $I(n, q)$ such that each $q$-tuple $\theta_{j}(1 \leqslant j \leqslant k)$ has $q$ distinct entries and $\{1, \ldots, q k\}$ is the disjoint union of these $k$ sets of entries. For each $\phi \in \Phi$ let $W_{\phi}$ be the subspace of $L^{k}\left(E_{n}^{\otimes q}\right)$ spanned by the elements $\left[e_{\theta_{1}}, \ldots, e_{\theta_{k}}\right]$ where $\left\{\theta_{1}, \ldots, \theta_{k}\right\}=\phi$. It follows that

$$
f_{q k}\left(L^{k}\left(E_{n}^{\otimes q}\right)\right)=\left(L^{k}\left(E_{n}^{\otimes q}\right)\right)^{\omega_{q k}}=\bigoplus_{\phi \in \Phi} W_{\phi}
$$

It is easily verified that $|\Phi|=(q k) ! / k !$. Also, by [MKS, Theorem 5.11], $\operatorname{dim} W_{\phi}=$ $(k-1)$ ! for all $\phi \in \Phi$. Thus

$$
\operatorname{dim} f_{q k}\left(L^{k}\left(E_{n}^{\otimes q}\right)\right)=(q k) !(k-1) ! / k !=(q k) ! / k .
$$

We consider $B^{r}(V)$, as in Section 2, in the special case where $G=\mathrm{GL}_{r}(F)$ and $V=E_{r}$ and we define

$$
C(r)=f_{r}\left(B^{r}\left(E_{r}\right)\right) .
$$

The following result will enable us to use $F \mathrm{GL}_{n}(F)$-modules for arbitrary values of $n$ with $n \geqslant r$.

Lemma 3.4. For all $n \geqslant r$, we have

$$
C(r) \cong f_{r}\left(B^{r}\left(E_{n}\right)\right) .
$$

Proof. We regard $E_{r}$ as a subspace of $E_{n}$ with $\mathrm{GL}_{r}(F) \leqslant \mathrm{GL}_{n}(F)$ in the obvious way. Let $d_{n, r}: \bmod _{F}(n, r) \rightarrow \bmod _{F}(r, r)$ be the truncation map (see [G, $\left.\S 6.5\right]$ ). For all $M \in \bmod _{F}(n, r)$, we have

$$
d_{n, r}(M)=\bigoplus_{\beta \in \Lambda(r, r)^{*}} M^{\beta}
$$

where $\Lambda(r, r)^{*}$ is the subset of $\Lambda(n, r)$ consisting of those weights $\left(\beta_{1}, \ldots, \beta_{n}\right)$ such that $\beta_{r+1}=\cdots=\beta_{n}=0$ (see [G, (6.5c)]). In particular, $f_{r}\left(d_{n, r}(M)\right)=f_{r}(M)$. It is a consequence of [BS1, Theorem 4.2] that $d_{n, r}\left(B^{r}\left(E_{n}\right)\right)=B^{r}\left(E_{r}\right)$. The result follows. 
By definition, $C(r)$ is a submodule of $\operatorname{Lie}(r)$. However, $B^{r}\left(E_{r}\right)$ is a direct summand of $E_{r}^{\otimes r}$ and, by [G, (6.3d)], $f_{r}\left(E_{r}^{\otimes r}\right)$ is a regular $F \mathfrak{S}_{r}$-module. Thus, by (3.2), $C(r)$ is a projective $F \mathfrak{S}_{r}$-module, and so $\operatorname{dim} C(r) \leqslant \operatorname{dim} \operatorname{Lie}^{\max }(r)$. Hence, in order to prove Theorem 1.2 , it suffices to prove that

$$
\lim _{\substack{r \rightarrow \infty \\ r \in \mathcal{A}}} \frac{\operatorname{dim} C(r)}{\operatorname{dim} \operatorname{Lie}(r)}=1 .
$$

For each $r \geqslant 1$, we define

$$
c_{r}=\frac{\operatorname{dim} C(r)}{\operatorname{dim} \operatorname{Lie}(r)}=\frac{\operatorname{dim} C(r)}{(r-1) !} .
$$

Thus $0 \leqslant c_{r} \leqslant 1$. It suffices to prove that $c_{r} \rightarrow 1$ as $r \rightarrow \infty$ with $r \in \mathcal{A}$.

Suppose that $m$ is a non-negative integer and $k$ is a positive integer such that $p \nmid k$ and $k \geqslant 2$. Take $n \geqslant r=p^{m} k$ and apply $f_{r}$ to the isomorphism (2.1) with $V=E_{n}$. By (3.2) we obtain

$$
\bigoplus_{i=0}^{m} p^{m-i} f_{r}\left(B^{p^{m-i} k}\left(E_{n}\right)^{\otimes p^{i}}\right) \cong f_{r}\left(L^{k}\left(E_{n}^{\otimes p^{m}}\right)\right) .
$$

By Lemma 3.2, Lemma 3.4 and (3.3), we have

$$
\operatorname{dim} f_{r}\left(B^{p^{m-i} k}\left(E_{n}\right)^{\otimes p^{i}}\right)=\frac{\left(p^{m} k\right) !}{\left(\left(p^{m-i} k\right) !\right)^{p^{i}}}\left(\operatorname{dim} C\left(p^{m-i} k\right)\right)^{p^{i}}=\frac{\left(p^{m} k\right) !}{\left(p^{m-i} k\right)^{p^{i}}}\left(c_{p^{m-i} k}\right)^{p^{i}} .
$$

Hence, by (3.4) and Lemma 3.3, we obtain

$$
\sum_{i=0}^{m} \frac{p^{m-i}\left(p^{m} k\right) !}{\left(p^{m-i} k\right)^{p^{i}}}\left(c_{p^{m-i} k}\right)^{p^{i}}=\frac{\left(p^{m} k\right) !}{k} .
$$

For $i=0,1, \ldots, m$, define

$$
a_{i}^{\prime}=\left(p^{m-i} k\right)^{-\left(p^{i}-1\right)} .
$$

Then (3.5) may be written as

$$
c_{p^{m} k}=1-\sum_{i=1}^{m} a_{i}^{\prime}\left(c_{p^{m-i} k}\right)^{p^{i}}
$$

Lemma 3.5. Suppose that $0 \leqslant s \leqslant i \leqslant m$. Then

$$
\frac{a_{i}^{\prime}}{a_{i-s}^{\prime}}=p^{-s}\left(\frac{p^{s}}{\left(p^{m-i} k\right)^{p^{s}-1}}\right)^{p^{i-s}} .
$$

Proof. We have

$$
\frac{a_{i}^{\prime}}{a_{i-s}^{\prime}}=\frac{\left(p^{m-i+s} k\right)^{p^{i-s}-1}}{\left(p^{m-i} k\right)^{p^{i}-1}}=\frac{\left(p^{s}\right)^{p^{i-s}-1}}{\left(p^{m-i} k\right)^{p^{i}-p^{i-s}}}=p^{-s}\left(\frac{p^{s}}{\left(p^{m-i} k\right)^{p^{s}-1}}\right)^{p^{i-s}}
$$

Proof of Theorem 1.2. Note the similarity between (3.6) and (2.3). Lemma 3.5 is stronger than Lemma 2.3 and therefore gives an analogue of Corollary 2.4. We do not need an analogue of Lemma 2.5 because the first term on the right-hand side of (3.6) is 1 . Thus, by the argument of the proof of Theorem 1.1, we obtain $c_{r} \rightarrow 1$ as $r \rightarrow \infty$ with $r \in \mathcal{A}$. 


\section{REFERENCES}

[BS1] R. M. Bryant and M. Schocker, 'The decomposition of Lie powers', Proc. London Math. Soc. (3) 93, 2006, 175-196.

[BS2] R. M. Bryant and M. Schocker, 'Factorisation of Lie resolvents', J. Pure Appl. Algebra 208, 2007, 993-1002.

[DE] S. Donkin and K. Erdmann, 'Tilting modules, symmetric functions, and the module structure of the free Lie algebra', J. Algebra 203, 1998, 69-90.

[ES] K. Erdmann and M. Schocker, 'Modular Lie powers and the Solomon descent algebra', Math. Z. 253, 2006, 295-313.

[ET] K. Erdmann and K. M. Tan, 'The Lie module of the symmetric group', Int. Math. Res. Not. IMRN 2010, Art. ID rnp244, 23 pages.

[G] J. A. Green, Polynomial Representations of $\mathrm{GL}_{n}$, Lecture Notes in Mathematics 830, second edition, Springer, Berlin, 2007.

[HB] B. Huppert and N. Blackburn, Finite Groups II, Springer, Berlin, 1982.

[J] G. D. James, The Representation Theory of the Symmetric Groups, Lecture Notes in Mathematics 682, Springer, Berlin, 1978.

[MKS] W. Magnus, A. Karrass and D. Solitar, Combinatorial Group Theory, second edition, Dover Publications, New York, 1976.

[R] C. Reutenauer, Free Lie Algebras, Clarendon Press, Oxford, 1993.

[SW1] P. Selick and J. Wu, 'On natural coalgebra decompositions of tensor algebras and loop suspensions', Mem. Amer. Math. Soc. 148, 2000, no. 701.

[SW2] P. Selick and J. Wu, 'Some calculations for Lie $(n)^{\max }$ for low n', J. Pure Appl. Algebra 212, 2008, 2570-2580.

(Roger M. Bryant) School of Mathematics, University of Manchester, Manchester M13 9PL, UK.

E-mail address: roger.bryant@manchester.ac.uk

(Kay Jin Lim and Kai Meng Tan) Department of Mathematics, National University of Singapore, Block S17, 10 Lower Kent Ridge Rodd, Singapore 119076.

E-mail address, K. J. Lim: matlkj@nus.edu.sg

E-mail address, K. M. Tan: tankm@nus.edu.sg 